\title{
Smart Constellation Selection for Precise Vehicle Positioning in Urban Canyons using a Software-Defined Receiver Solution
}

\author{
Brian Niehoefer, Florian Schweikowski and Christian Wietfeld \\ Communication Networks Institute \\ TU Dortmund University \\ Dortmund, Germany \\ brian.niehoefer|florian.schweikowski|christian.wietfeld@tu-dortmund.de
}

\begin{abstract}
Positioning techniques based on satellite navigation systems are nowadays standard in most commercial vehicles. But GPS receivers in general are effected by multipath and atmospheric effects and hence their performance may be decreased, especially in challenging environments that directly affects the usage of satellite-based positioning in traffic-relevant and by that safety-critical systems.

Therefore, this paper describes, based on an exemplary implementation of an Advanced Software-Defined radio receiver (ASDR) for Global Navigation Satellite Systems, the impact of the direct receiver surrounding. In addition, a socalled Smart Constellation Selection (SCS) as pre-positioning routine will be introduced to increase the accuracy by just using already available data at the receiver side. Next to the implementation of the ASDR itself, the functionality and performance of the SCS will be explained. In addition, this contribution contains the introduction of a freely available database with geo-referenced GPS measurements including web-based analysis functionalities to enable other researches to test and evaluate their ideas for future GNSS enhancements.
\end{abstract}

Keywords-Satellite Positioning; Software-Defined GNSS Receiver; SDR; Positioning Enhancement; Resource-efficiency;

\section{INTRODUCTION}

Reliable and accurate positioning is a well known major key performance indicator within traffic-relevant and by that safety-critical systems, like Driving Assistance, Green Car Mobility or Traffic Manipulation. During the past years, techniques using the Global Positioning System (GPS), as actual part of the future Global Navigation Satellite System (GNSS), have established as a standard solution for positioning tasks in outdoor scenarios. But although the actual guaranteed accuracy is sufficient for the typical every-day applications, the overall quality of the positioning cannot be assumed in general [9] [11]. In dense urban areas or other challenging environments, the positioning accuracy may decrease dramatically, caused by blocked or reflected GNSS signals [12]. Figure 1 visualizes one of the main remaining scientific challenges in GNSS positioning, the so-called 'urban canyons'. Several obstacles may block or influence GNSS signals incoming from nearly the same direction, while other directions, e.g. along a street, provide a free Line-of-Sight (LOS). This ends up in a poor position

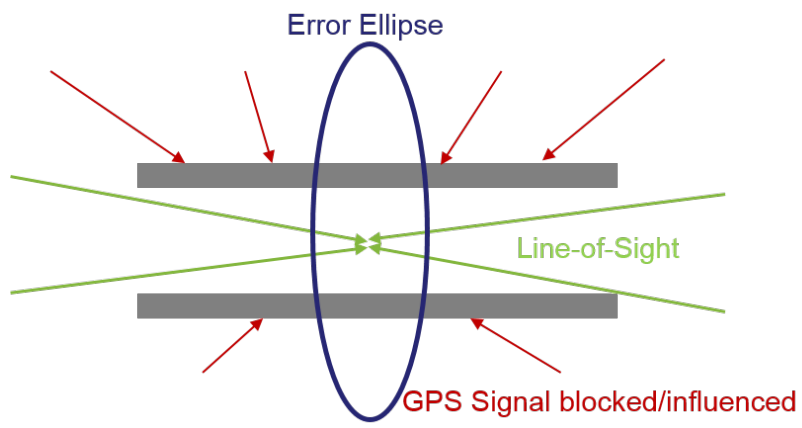

Figure 1. Urban Canyons as Scientific Challenge for GPS [4]

solution geometry (low Dilution of Precision, DOP) along, and high DOP across the street and by that, a much lower accuracy in the cross-street direction [4].

Hence, the Collaborative Research Center 876, sub-project B4 as well as the research project AIRBEAM focus on the compensation of such effects to reach a positioning within $1.5 \mathrm{~m}$ using satellite navigation systems. Hereby the sub-project B4 mainly faces the lane-specific positioning of traffic objects (so $<1.5 \mathrm{~m}$ ) for traffic predictions. The project AIRBEAM also may take advantage of an improved and predictable positioning accuracy to improve the mobility routine of Unmanned Aerial Vehicles (UAVs) within emerging situations. Former publications already show the influences caused by atmospheric or local effects, like multipath or shadowing, using a simulation framework including ray tracing [8][9]. To confirm these results and to enable a separated analysis of all influences, this contribution deals with the development of an Advanced Software-Defined GNSS Receiver (ASDR) based on GNU Radio. Next to the analysis of all impact components, the provided solution allows the comparison and performance evaluation of different existing models, like UNB3 or Klobuchar. In addition, the implemented Dual-Link characteristic enables a data-exchange using web-services for actual and future compensation methods. At the end, this contribution introduces a web-based and freely available database providing 
several unmodified GPS measurements, in our case used as exemplary GNSS system, on two geo-referenced spots. Any interested user can download this raw data, perform functions and improvements, and upload the results back to the web-service to evaluate the gained performance using the integrated multiple analysis methods.

This contribution is structured as follows: Before a detailed explanation of our approach in Section III, Sections II provides a short overview about Software-Defined GNSS receivers and ongoing research approaches to increase the accuracy of GNSS systems. In Section IV the benefits of this contribution to the Global Navigation Satellite System are highlighted, before Section V wraps up with a conclusion.

\section{RESEARCH TOPICS ON SOFTWARE-DEFINED GNSS RECEIVERS AND ADVANCED GNSS POSITIONING TECHNIQUES}

To delimit and grade this contribution, the upcoming section will give a short overview about the actual research topics and ideas in this field of application.

\section{A. Functionalities, Benefits and Ongoing Work of Software- Defined Radios within GNSS}

Software Defined Radio (SDR) might be understood as a platform for the introduction of new technologies and services into existing live networks. It extends well beyond the simple configuration of air interface parameters to cover the whole system from the network to service creation and application development [1]. Thereby, the key idea of the SDR design paradigm can be described by three main advantages: Reconfigurability, Updatability and Flexibility [13].

Especially when thinking about multiple satellite positioning systems combined to a GNSS this adaptability clarifies the effectiveness and the advantage of using SDRs for satellite-based positioning techniques. Hence, based on the general functions and setups of SDRs, which are explained in [1], a Software-Defined Radio adapted to receive and analyze GNSS signals is an obvious improvement. Different ideas like Software-Controlled Hardware Architecture, Fully Software-Controlled or Hybrid Approaches are explained in [2] and [14] but those are focusing the GNSS functionality itself, without any extensions or GNSS performance analyses or improvements. The same holds true for applicationspecific research projects like [5]. Hereby, a SDR approach will be developed, which enables a hardware-independent control and steering using highly precise GPS positioning. But again, although an SDR approach is used, the outcome of this work is limited to this kind of application and will not allow any further outcome to other research projects. In contrast, this contribution analyzes different nowadays compensation models and their impact to the positioning, integrated former outsourced filtering routines in GNU Radio and also addresses resource-efficient accuracy enhancements in the front-end itself.

\section{B. Actual Research on Advanced GNSS-based Positioning}

Inaccuracies within satellite positioning techniques, especially in urban canyons (introduced in Section I) are well-known in the scientific field. Hence, there are several solution approaches, whereby the main research direction focuses on the identification and exclusion/weighting of NonLine-of-Sight (NLOS) signal paths. One realization method is the use of additional hardware like antenna arrays [6] or sky-pointing cameras [7]. Both approaches check the LOS reachability for each received satellite using their roughly estimated position and the satellite's ephemeris data or a dynamic generated elevation mask using the camera respectively. But although both methods show a significant performance gain, they also do increase cost, size and power consumption of the user equipment and are not usable with nowadays well-known and widespread GPS equipment.

Other generally valid approaches take advantage of $3 \mathrm{D}$ models to assist the positioning routines. E.g. [4] uses them to calculate the probability of receiving a GPS signal directly, the so-called Shadow Matching. [11] takes the same idea, but uses ray tracing technology to identify the NLOS signals. But all of these approaches are missing either the general validity or they depending on a reliable inertial positioning, what cannot be assured especially in challenging environments.

In contrast, the so-called Smart Constellation Selection (SCS) presented in this paper, takes advantage of available measurements within the GPS receiver and tries to evaluate the best-case satellite constellation of those signals received, without additional hardware or complex back-end computing routines. In addition, the developed ASDR combined with web-based evaluation routines allows a quantification of the gained performance and addresses other researchers to do so using the gained and provided geo-referenced results.

\section{AdVANCEd SOFTWARE-DEFINED GNSS RECEIVER - DIFFERENCES AND BENEFITS}

As described in Section II, Software-Defined GNSS receivers are already known in the scientific world. Hence this section will just give a short overview about the positioning routine for SDRs and differences of the implementation for this contribution.

\section{A. SD-GNSS Architecture}

The developed SD-GNSS receiver is based on a 2 nd generation USRP from Ettus Research. To guarantee the reception of GPS and Glonass signals the authors add an L1 antenna and an amplifier from AuCon with an overall gain of $65 \mathrm{~dB}$. Beneath the signal reception itself, the SD-GNSS functionalities, implemented on the device using GNU Radio may be separated into six steps, concerning to [3], and were integrated in Figure 2 on the right and left side.

Based on the low amplitude of GNSS signals, a Filtering step is necessary to extract the satellite signals from the 
background noise. The Acquisition uses the correlation of the recorded, filtered signal sequence with the one knew from (in this case) GPS and calculates the maxima. Based on the low signal strength and different influences from atmosphere and the direct receivers surrounding, the acquisition itself is error-prone and might tend to fail-acquisitions in challenging environments. All identified reachable satellite signals are then forwarded in the so-called Acquisition Matrix in the order of the best correlation to the Tracking routine. Hereby the analysis of code and phase of the signal in interacting loops (Phase Lock Loop and Delay Lock Loop) for each satellite signal increases the accuracy performance and by that allows a positioning within a few meters. The following Error Estimation calculate and minimize known failures, like atmospheric ones based on different models, deviations in the receiver's clock or the satellite orbits. The last step then uses the least squares algorithm to extract the most probable position.

\section{B. Advanced SD-GNSS Receiver (ASDR)- Improvements and Differences}

Next to the basic functionalities of GNSS receiver implementations for SDRs, this contribution also addresses some modifications and improvements respectively, which are visualized in Figure 2 and which will be explained in the upcoming section.

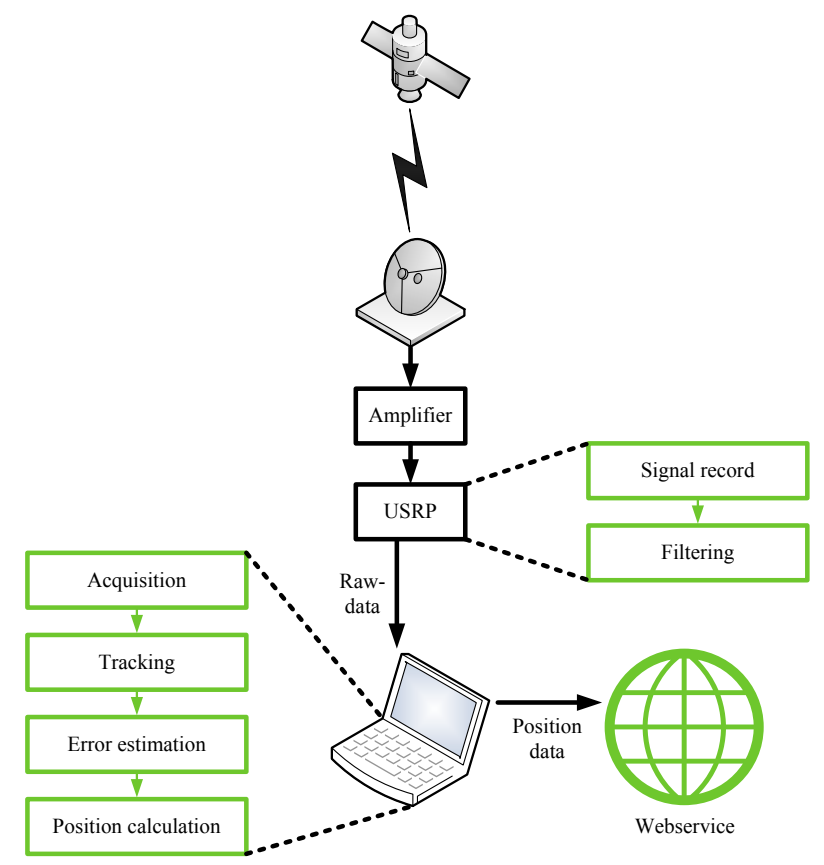

Figure 2. Functionalities of the Advanced Software-Defined GNSS Receiver

First of all, the filtering routine was integrated within the GNU Radio implementation. This comes along with the advantage to monitor the filtering steps during run- time in the field. A further modification addresses a duallink architecture to allow back-end applications to increase the positioning performance via post-processing and send those back to the SD-GNSS receiver. In addition, the socalled Smart Constellation Selection (SCS) uses known circumstances and measurements in the receiver to increase the positioning performance without additional hardware or resource-intensive algorithms or communication.

1) Stand-Alone Filtering Mechanics: Nowadays GNSS receivers based on Software-Defined radios are mostly separated into a heterogeneous bunch of calculating entities. The SDR normally is used to receive the signal itself, whereby an ordinary PC is used for the filtering (e.g. audio filter programs like avidemux) [2]. To simplify this procedure, and nevertheless to keep the equipment portable and to be able to monitor the success of detecting the satellites during the recording of the data-stream, the filtering routine (shown in Figure 3) was integrated into the SDR functionalities. Instead of using an ordinary low-pass filter, the ASDR uses an interpolating Finite Impulse Response (FIR) filter with low-pass properties to enable a variable sampling rate later on. Because the USRP always transforms received signals into the baseband, the I and Q parts are separated using orthogonal superimpositions into the Intermediate Frequency (IF) and reassembled afterwards for the Soft GNSS routine as processable data stream containing all phase information.

2) Dual-Link Characteristic: Beside different performance improvements within the ASDR itself, it is an irrevocable fact that an ASDR is an embedded and by that a resource constrained device, making complex calculations nearly impossible without neglecting other KPIs. Hence, the authors added an independent communication link to allow back-end server solutions to improve the result accuracy afterwards.

For this contribution, the authors used a second SDR and near-field communication to simulate the data-exchange between the ASDR and the developed web-service. The idea is to store the data with the calculated GPS/GNSS position, recalculate the result using compensation or filtering routines, like explained in [8] and [9], and provide it back to the accessible web-server. To clarify the proper functioning and to gain further scientific benefits for the community, the authors start with the upload of geo-referenced GPS measurements to allow further researchers to test and validate their ideas to improve the GNSS positioning accuracy (see Section IV-B).

3) Smart Constellation Selection (SCS) -Known Circumstances, Predictable Effects: The main idea of the SCS is to take advantage of known circumstances which indicate a potential negative impact on the accuracy. It turns out, that the exclusion of certain satellite signals in the positioning routine may increase the arising overall performance in some cases. One of these factors is the respectively given receiver-satellite geometry, the so-called Geometric Dilution 


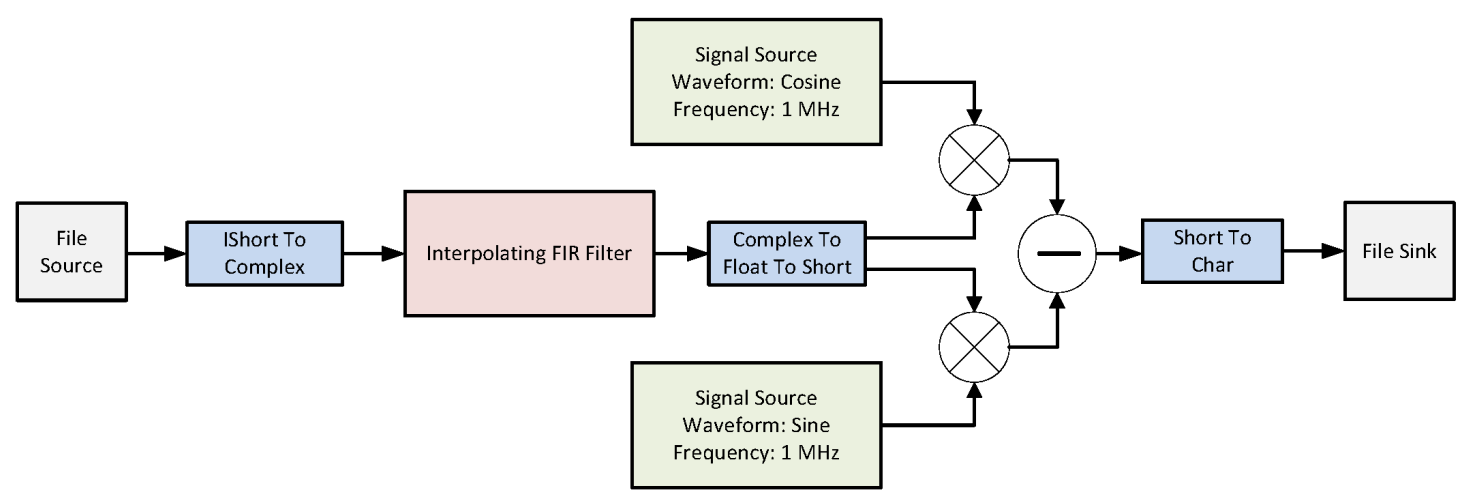

Figure 3. Stand-Alone Filter Mechanism implemented in GNU Radio

of Precision (GDOP). The GDOP may be described as the size of the potential positioning area. So a high GDOP indicates a high probability of incorrect positioning.

The main task is to predict those impacts by just using the already existing values at the receiver side. Of course, the GDOP itself just may be used to evaluate the actual given constellation, so the approach is, as long as there are more than the minimum four satellites in range, to analyze every possible combination of all received satellite signals. To compare those combinations and to figure out which one of those is the most qualified, the authors suggest the following rating equation, which is derived by empirical measurements using the ASDR:

$$
S C S_{\text {Rating }}=G D O P_{\text {diff }} \cdot h_{\text {AcqMetric }} \cdot \frac{n}{p}
$$

In this case $n$ is the number of the overall amount of satellites, $p$ the position of the actual excluded one in the acquisition metric (comp. Section III-A), $h_{\text {AcqMetric }}$ for its acquisition value and $G D O P_{\text {diff }}$ the difference between the origin GDOP value (taking all satellites in range) and the appropriate GDOP value after the exclusion. All of them are obvious factors, because a good acquisition value indicates high reduction of the overall performance when excluding the signal. The same holds true for a comparable high GDOP increase. This rating is performed for every possible satellite combination, whereby the lowest value at the end indicated the best performing constellation. If all ratings are $>2$, no promising improvement can be recommended.

The main advantage of this approach is the resourceefficiency in terms of computation time and energy consumption. All used indicators are already known at the receiver side and the rating procedure is not too complex. The disadvantage is of course, that there is a need to receive more than the necessary four satellites to perform this kind of analysis, what is not always the case in the actual GPS constellation. Referring to the increasing amount of receivable satellites in the upcoming GNSS constellation (GPS + Glonass + Galileo), the probability will increase in the next few years. The positioning improvement of the SCS itself, will be explained in Section IV-C.

\section{ENHANCEMENTS AND BENEFITS TO GLOBAL Navigation Satellite System}

\section{A. Real-World Quantification of different Compensation Models}

As described in Section II, there are different parameters included within the GPS signal to compensate atmospheric influences, e.g. for the troposphere and ionosphere which both have a significant impact to satellite signals and by that to the positioning accuracy. The advantage of the developed ASDR is that both, the errors and corresponding compensation techniques, can be determined and by that, may be compared in the future. Figure 4 visualizes one of these comparisons. In this case the positioning errors derived

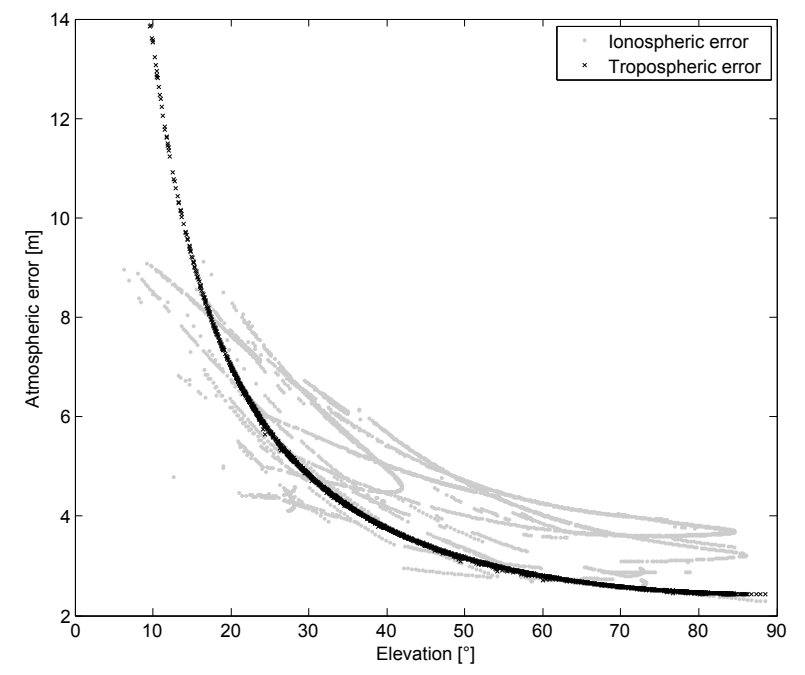

Figure 4. Comparison of Tropospheric and Ionospheric Compensation Methods

from two compensation models, UNB3 for the troposphere and Klobuchar for the ionosphere, are plotted depending on 
the specifically given elevation angle. It is obvious, that the UNB3 model shows negative exponential behavior with a given parameter set, apposite to the more static influences of the troposphere. The Klobuchar model on the other hand is more difficult to model and therefore based on actual measurements and by that is more accurate and adaptable.

\section{B. Open-Source Geo-referenced GPS Measurements and Statistics}

Before clarifying the performance of the SCS itself, this contribution address a further outcome. To enable a reliable evaluation of GNSS positioning techniques, the existence of geo-referenced points as reference for the measurement equipment is mandatory. Hence, the authors uses two of these points specified by the land surveying office at the campus of the university in Dortmund, to quantify the performance gain of the SCS. All measurements were performed at these two points and were stored in a database which is connected to a web-service to enable an easy handling and analysis with the included MapView (comp. Figure 5) of all measured points, a TabularView with a detailed listing of all recorded properties per point (e.g. corresponding time, satellite constellation, GDOP) and Analyzing/Static tools to evaluate certain recorded points.

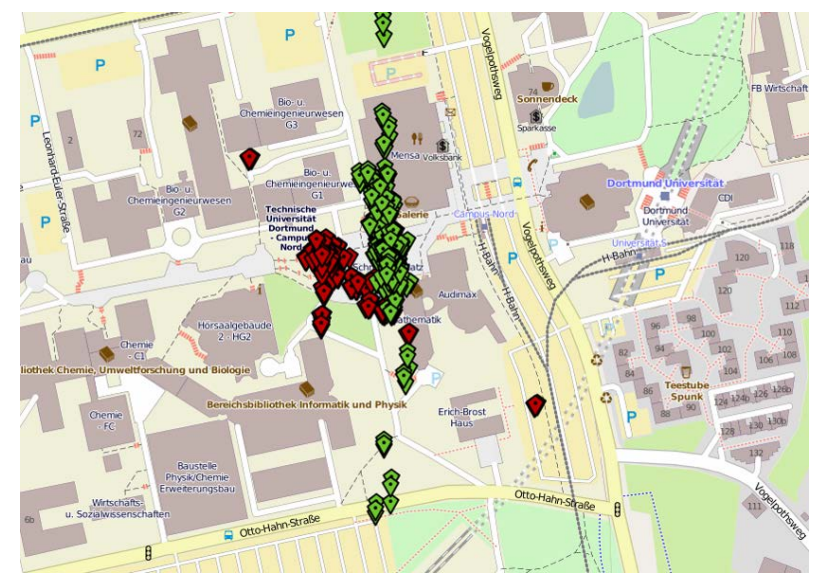

Figure 5. Web-Database and GNSS Evaluation Tool

The idea is to freely provide this content to any scientist in the world to enable and validate any position correcting algorithm.

\section{Performance of the Smart Constellation Selection}

Section III-B3 already clarified the advantages of the SCS: resource-awareness, simplicity and direct practicability. Based on the empirical derivation, the gained rating (comp.- Formula 1) cannot be generally validated and there will always be some cases where SCS may under-perform. Hence, it is necessary to quantify the amount of cases where SCS can be used to increase the positioning accuracy and to

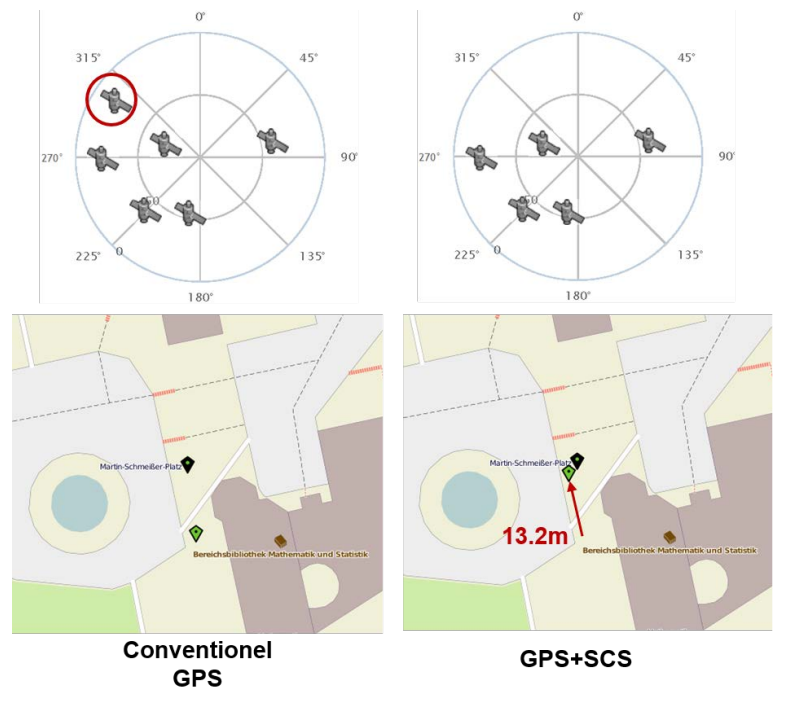

Figure 6. Exemplary Performance Gain using SCS

analyze its contribution. One exemplary performance gain of the SCS is shown in Figure 6.

The top of the figure shows the visualization derived by the Open Source Satellite Simulator [10] of two used and recorded constellations for the ASDR positioning routine, whereby the differences is just the exclusion of one satellite. The performance gain of this procedure is shown on the bottom of the figure, recorded and analyzed using the ASDR. By just ignoring the satellite marked with a red circle (identified by the SCS), the positioning performance increases by $13.2 \mathrm{~m}$.

But the case described above is just one snapshot and cannot be used to outline and guarantee the SCS performance. So, in a further step, the authors analyze more than 500 GPS recordings on two reference points near the campus of the Dortmund, TU University (comp. Section IV-B). Both points are matching to the definition of the introduced urban canyons and by that, standing for the most challenging environments for GNSS. The authors chose these locations to ensure a worst-case consideration, meaning that in a 'normal' GNSS situation there should be even more satellites visible and by that the performance of the SCS will enhance based on the increased number of possible constellations for the SCS. Nevertheless, more than $15 \%$ of all measurements indicated an increased performance when using the SCS. Figure 7 visualizes the results as a violin plot, so the kernel density (green envelope), .25 and .75 quantiles (rectangle), mean value (white dot) and upper/lower adjacent value (black line) are directly visible. The positioning error decreases in all mentioned terms when using the SCS, even in direct comparison to the included atmospheric models. In case of the mean value an improvement of more than $1.5 \mathrm{~m}$ and, even more important, the lower peak value clarifies 


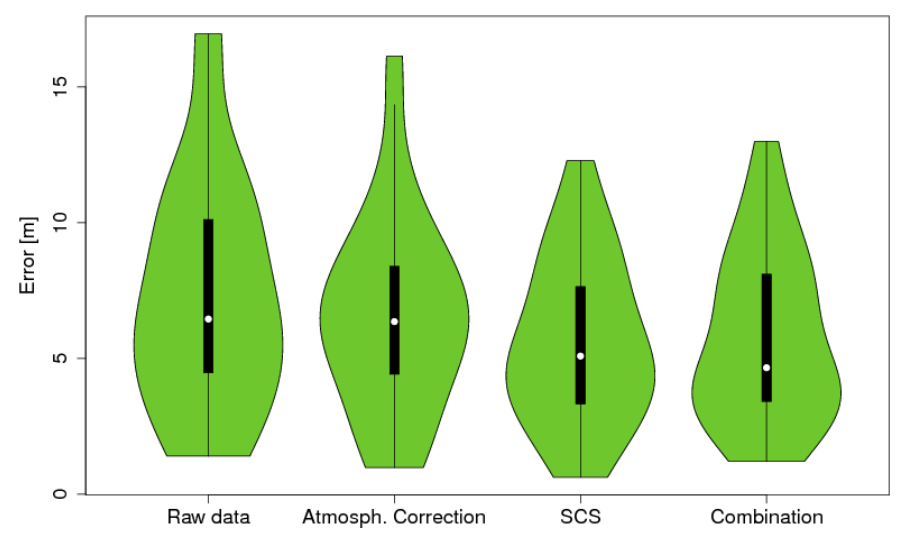

Figure 7. Overall Performance of the SCS

the benefit of using SCS. Again, it should be mentioned that SCS is based on already known measurements and information at the receiver side, without additional hardware or complex calculation what increases the relevance of SCS in the authors opinion even more. Figure 7 also visualize the combination of the SCS with the GPS internal atmospheric correction, which shows again a slight performance increase, referring to the mean value. The higher peak values are caused by partially mis-compensations determined by the GPS internal atmospheric model.

\section{CONCLUSion}

In this paper the authors described the development, functionalities and benefit of an Advanced Software-Defined GNSS Receiver (ASDR). Based on the ASDR, a resourceaware Smart Constellation Selection (SCS) was introduced, which uses already available information at the receiver to identify potential performance enhancements of up to $13 \mathrm{~m}$ when excluding certain satellites in some cases. The overall performance of SCS was analyzed using two reference points and estimated to more than $1.5 \mathrm{~m}$ on average without any additional hardware or complex calculation. In addition, a freely available web-service was presented, to provide the recorded measurements and multiple analyzing tools to other scientists, so that they can analyze and estimate their positioning enhancements in the future.

\section{ACKNOWLEDGMENT}

The work on this paper has been partially founded by Deutsche Forschungsgemeinschaft (DFG) within the Collaborative Research Center SFB 876 "Providing Information by Resource-Constrained Analysis", project B4 and by the European Union Seventh Framework Programme (FP7/20072013) under grant agreement 261769.

\section{REFERENCES}

[1] M. Dillinger, K. Madani, and N. Alonistioti. Software defined radio: Architectures, systems and functions. Wiley. com, 2005.
[2] G. Girau, A. Tomatis, F. Dovis, and P. Mulassano. Efficient software defined radio implementations of gnss receivers. In Circuits and Systems, 2007. ISCAS 2007. IEEE International Symposium on, pages 1733-1736, 2007.

[3] P. D. Groves. Principles of GNSS, inertial, and multisensor integrated navigation systems. 2008.

[4] P. D. Groves, Z. Jiang, L. Wang, and M. K. Ziebart. Intelligent urban positioning using multi-constellation gnss with $3 \mathrm{~d}$ mapping and nlos signal detection. Proc. ION GNSS, 2012.

[5] M. Ilg. Framework for a software-defined global positioning system (gps) receiver for precision munitions applications. Technical report, DTIC Document, 2012.

[6] M. Keshvadi, A. Broumandan, and G. Lachapelle. Analysis of gnss beamforming and angle of arrival estimation in multipath environments. In Proceedings of the Institute of Navigations 2011 International Technical Meeting (ION ITM), San Diego, CA, USA, pages 24-26, 2011.

[7] J. Meguro, T. Murata, J. Takiguchi, Y. Amano, and T. Hashizume. Gps multipath mitigation for urban area using omnidirectional infrared camera. Intelligent Transportation Systems, IEEE Transactions on, 10(1):22-30, 2009.

[8] B. Niehoefer, S. Lehnhausen, and C. Wietfeld. Combined Analysis of Local Ionospheric and Multipath Effects for Lane-Specific Positioning of Vehicles within Traffic Streams. In 6th ESA Workshop on Satellite Navigation Technologies (NaviTech), Noordwijk, The Netherlands, December 2012.

[9] B. Niehoefer, A. Lewandowski, and C. Wietfeld. Evaluation of the Localization Accuracy of Satellite Systems for Traffic Flow Predictions. In 24th International Technical Meeting of The Satellite Division of the Institute of Navigation (ION GNSS 2011), Portland, Sep 2011.

[10] B. Niehoefer, S. Subik, and C. Wietfeld. The cni open source satellite simulator based on omnet++. In OMNeT++ Workshop 2013 - 6th International Workshop to be held in conjuntion with SIMUTools, 2013.

[11] M. Obst, S. Bauer, and G. Wanielik. Urban multipath detection and mitigation with dynamic $3 \mathrm{~d}$ maps for reliable land vehicle localization. In Position Location and Navigation Symposium (PLANS), 2012 IEEE/ION, pages 685-691. IEEE, 2012.

[12] M. Obst, N. Mattern, R. Schubert, and G. Wanielik. Car-tocar communication for accurate vehicle localizationthe covel approach. In Systems, Signals and Devices (SSD), 2012 9th International Multi-Conference on, pages 1-6. IEEE, 2012.

[13] F. Principe, G. Bacci, F. Giannetti, and M. Luise. Softwaredefined radio technologies for gnss receivers: A tutorial approach to a simple design and implementation. International Journal of Navigation and Observation, 2011, 2011.

[14] J. Tian, W. Ye, S. Lin, and Z. Hua. Software defined radio gnss receiver design over single dsp platform. In Wireless Communications, Networking and Mobile Computing, 2008. WiCOM '08. 4th International Conference on, pages 1-4, 2008. 